DESY 04-197

Edinburgh 2004/25

Leipzig LU-ITP 2004/023

Liverpool LTH 637

\title{
Perturbative Renormalisation of the Second Moment of Generalised Parton Distributions
}

\author{
M. Göckeler ${ }^{1,2}$, R. Horsley ${ }^{3}$, H. Perlt ${ }^{2,1}$, P. E. L. Rakow ${ }^{4}$, A. Schäfer ${ }^{2}$, \\ G. Schierholz ${ }^{5,6}$ and A. Schiller ${ }^{1}$ \\ ${ }^{1}$ Institut für Theoretische Physik, Universität Leipzig, \\ D-04109 Leipzig, Germany \\ ${ }^{2}$ Institut für Theoretische Physik, Universität Regensburg, \\ D-93040 Regensburg, Germany \\ ${ }^{3}$ School of Physics, University of Edinburgh, Edinburgh EH9 3JZ, UK \\ ${ }^{4}$ Theoretical Physics Division, Department of Mathematical Sciences, \\ University of Liverpool, Liverpool L69 3BX, UK \\ ${ }^{5}$ John von Neumann-Institut für Computing NIC, \\ Deutsches Elektronen-Synchrotron DESY, D-15738 Zeuthen, Germany \\ ${ }^{6}$ Deutsches Elektronen-Synchrotron DESY, D-22603 Hamburg, Germany
}

\begin{abstract}
We calculate the non-forward quark matrix elements of operators with two covariant derivatives needed for the renormalisation of the second moment of generalised parton distributions in one-loop lattice perturbation theory using Wilson fermions. For some representations of the hypercubic group commonly used in simulations we determine the sets of all possible mixing operators. For those representations the one-loop mixing matrices of renormalisation factors are found. Due to non-vanishing contributions of operators with external ordinary derivatives the number of contributing operators increases compared to forward matrix elements.
\end{abstract}




\section{Introduction}

In recent years generalised parton distributions (GPDs) [1] became a focus of both experimental and theoretical studies in hadron physics. For an extensive up-to-date review including a comprehensive list of references see [2]. GPDs provide a universal (with the meaning used in factorisation proofs), unifying parametrisation for a large class of hadronic correlators, including e.g. form factors and the ordinary parton distribution functions. Thus they provide a solid formal basis to connect information from various inclusive, semi-inclusive and exclusive reactions in an efficient, unambiguous manner. Furthermore they give access to physical quantities which cannot be directly determined in experiments, like e.g. the orbital angular momentum of quarks and gluons in a nucleon (for a chosen specific scheme) and the spatial distribution of the energy or spin density of a fast moving hadron in the transverse plane. This enormous potential motivates the ongoing dedicated investigation of exclusive reactions at DESY, CERN, JLab and other accelerator centers [3, 4]. As GPDs are well-defined QCD objects it was possible to derive many fundamental theoretical results, e.g. the form of their NLO- $Q^{2}$-evolution equations as well as the NLO coefficient functions for Deeply Virtual Compton Scattering.

However, the direct experimental access to GPDs beyond the limiting cases of distribution functions and simple form factors is limited. So one can at most hope to compare experimental data with suitable parametrisations or models. But even under the most optimistic assumptions one would certainly need of the order of 20 parameters per flavour for a reliable fit of all GPDs. (Basically, because GPDs contain so much physics they cannot be expected to be trivial functions.) Another practical problem is that exclusive cross sections typically fall so rapidly with $Q^{2}$ that only moderate $Q^{2}$ values can be studied. For these, however, higher-twist contributions can be sizeable, which complicates the situation even further.

Therefore, although there is in principle an enormous number of reaction channels which provide information on GPDs, in practice it is indispensable to obtain complementary information, e.g. from lattice QCD calculations. On the lattice we can compute

matrix elements of local composite operators, and moments of GPDs can be related to such matrix elements taken between states of different nucleon momenta and spins. 
More precisely we can write [5] for example

$$
\begin{aligned}
\left\langle p^{\prime}\left|\mathcal{O}_{\mu_{1} \cdots \mu_{n}}\right| p\right\rangle & =\bar{u}\left(p^{\prime}\right) \gamma_{\left(\mu_{1}\right.} u(p) \sum_{i=0}^{\left[\frac{n-1}{2}\right]} A_{n, 2 i}(t) \Delta_{\mu_{2}} \cdots \Delta_{\mu_{2 i+1}} \bar{p}_{\mu_{2 i+2}} \cdots \bar{p}_{\left.\mu_{n}\right)} \\
& -\frac{1}{2 M} \bar{u}\left(p^{\prime}\right) i \Delta^{\alpha} \sigma_{\alpha\left(\mu_{1}\right.} u(p) \sum_{i=0}^{\left[\frac{n-1}{2}\right]} B_{n, 2 i}(t) \Delta_{\mu_{2}} \cdots \Delta_{\mu_{2 i+1}} \bar{p}_{\mu_{2 i+2}} \cdots \bar{p}_{\left.\mu_{n}\right)} \\
& +C_{n}(t) \operatorname{Mod}(n+1,2) \frac{1}{M} \bar{u}\left(p^{\prime}\right) u(p) \Delta_{\left(\mu_{1}\right.} \cdots \Delta_{\left.\mu_{n}\right)},
\end{aligned}
$$

where all indices are symmetrised and trace terms are subtracted as indicated by $(\cdots)$. The leading twist-two operators used in (11) are

$$
\mathcal{O}_{\mu_{1} \cdots \mu_{n}}=\left(\frac{i}{2}\right)^{n-1} \bar{\psi} \gamma_{\left(\mu_{1}\right.} \stackrel{\leftrightarrow}{D}_{\mu_{2}} \cdots \stackrel{\leftrightarrow}{D}_{\left.\mu_{n}\right)} \psi
$$

with the symmetric covariant derivative

$$
\stackrel{\leftrightarrow}{D}=\vec{D}-\overleftarrow{D}
$$

Analogous equations exist for

$$
\mathcal{O}_{\mu_{1} \cdots \mu_{n}}^{5}=\left(\frac{i}{2}\right)^{n-1} \bar{\psi} \gamma_{\left(\mu_{1}\right.} \stackrel{\leftrightarrow}{D}_{\mu_{2}} \cdots \stackrel{\leftrightarrow}{D}_{\left.\mu_{n}\right)} \gamma_{5} \psi
$$

and for the tower of operators involving $\sigma_{\mu \nu}$ (related to the generalised transversity) [6]. In (1I) we have the nucleon 4-momentum transfer $\Delta=p^{\prime}-p$ and its invariant $t=\Delta^{2}$, $\bar{p}=\left(p^{\prime}+p\right) / 2$ denotes the average nucleon momentum and $M$ its mass.

The generalised form factors $A_{n, 2 i}(t), B_{n, 2 i}(t)$ and $C_{n}(t)$ are related to the moments of the GPDs by ${ }^{1}$

$$
\begin{aligned}
\int_{-1}^{1} d x x^{n-1} H(x, \xi, t) & =\sum_{i=0}^{\left[\frac{n-1}{2}\right]} A_{n, 2 i}(t)(-2 \xi)^{2 i}+\operatorname{Mod}(n+1,2) C_{n}(t)(-2 \xi)^{n}, \\
\int_{-1}^{1} d x x^{n-1} E(x, \xi, t) & =\sum_{i=0}^{\left[\frac{n-1}{2}\right]} B_{n, 2 i}(t)(-2 \xi)^{2 i}-\operatorname{Mod}(n+1,2) C_{n}(t)(-2 \xi)^{n} .
\end{aligned}
$$

\footnotetext{
${ }^{1}$ As an example we give here the off-forward parton distributions $H(x, \xi, t)$ and $E(x, \xi, t)$, one of which reduces to the ordinary quark distribution in the limit $\xi \rightarrow 0$ and $t \rightarrow 0: H(x, 0,0)=q(x)$.
} 
The variable $\xi=-n \cdot \Delta / 2$ is defined with the help of a light-like vector $n^{\mu}$ which obeys $\bar{p} \cdot n=1$.

First results for moments of GPDs obtained on the lattice were published recently [7, 8] (see also [9]) and soon results from improved calculations should become available. Not surprisingly, a number of theoretical problems still has to be settled. One urgent task is to obtain the missing renormalisation factors and to completely analyse the operator mixing. The renormalisation of the operators which are related to (generalised) parton distributions has been discussed extensively, both in the continuum and on the lattice. Up to now, however, almost exclusively the case of forward matrix elements has been considered. When non-forward matrix elements are studied, new features arise, which make a reconsideration of the renormalisation problem necessary. In particular, the mixing with "external ordinary derivatives", i.e. with operators of the form $\partial_{\mu} \partial_{\nu} \cdots(\bar{\psi} \cdots \psi)$, needs to be investigated [11. They do not contribute in forward matrix elements, but have to be taken into account when calculating e.g. the generalised form factors $A_{n, 2 i}(t)$ for $i>0$.

On the lattice the mixing patterns are usually more complicated than in the continuum, because covariance under the hypercubic group $H(4)$ imposes less stringent restrictions than $O(4)$ covariance. The necessity to consider also operators with external ordinary derivatives enlarges the set of contributing operators even further. These complications do not yet arise for $n=1$ and $n=2$. Hence for these moments the renormalisation factors can be taken over from the forward case.

In this paper we investigate the renormalisation problem for $n=3$ within the framework of one-loop lattice perturbation theory. Some first results have been presented recently [10]. We find that the numerical values of the renormalisation factors are not very different from one. We also find that the pattern of operator mixing is far more involved than e.g. for moments of the ordinary parton distributions. While the numerical results from one-loop lattice perturbation theory have limited precision, the results concerning the mixing of operators are valid in general. Note also that our considerations apply equally well to moments of distribution amplitudes.

Let us fix the notations used in our perturbative calculations. We work in Euclidean space and use the Wilson gauge action and Wilson fermions such that the total action is given by

$$
S_{\mathrm{W}}^{\text {latt }}=S_{\mathrm{W}, \mathrm{F}}+S_{\mathrm{W}, \mathrm{G}} .
$$


The fermionic part $S_{\mathrm{W}, \mathrm{F}}$ for dimensionful massless fermion fields $\psi(x)$ has the form

$$
\begin{aligned}
S_{\mathrm{W}, \mathrm{F}} & =4 a^{3} r \sum_{x} \bar{\psi}(x) \psi(x) \\
& -\frac{a^{3}}{2} \sum_{x, \mu}\left[\bar{\psi}(x)\left(r-\gamma_{\mu}\right) U_{x, \mu} \psi(x+a \hat{\mu})+\bar{\psi}(x+a \hat{\mu})\left(r+\gamma_{\mu}\right) U_{x, \mu}^{\dagger} \psi(x)\right],
\end{aligned}
$$

where $a$ is the lattice spacing and the sums run over all lattice sites $x$ and directions $\mu$ on the lattice (all other indices are suppressed). The link matrices $U_{x, \mu}$ are related to the gauge field $A_{\mu}(x)$ by

$$
U_{x, \mu}=\exp \left[i g a A_{\mu}(x)\right], \quad A_{\mu}(x)=T^{c} A_{\mu}^{c}(x),
$$

where $g$ is the bare gauge coupling and the $T^{c}$ are the generators of the $S U(3)$ algebra. The Wilson parameter $r$ can be chosen from the interval $(0,1]$. The gauge action for the gluon field $A_{\mu}(x)$ is

$$
S_{\mathrm{W}, \mathrm{G}}=\frac{6}{g^{2}} \sum_{x, \mu<\nu}\left[1-\frac{1}{6} \operatorname{Tr}\left(U_{x, \mu \nu}+U_{x, \mu \nu}^{\dagger}\right)\right]
$$

with

$$
U_{x, \mu \nu}=U_{x, \mu} U_{x+a \hat{\mu}, \nu} U_{x+a \hat{\nu}, \mu}^{\dagger} U_{x, \nu}^{\dagger}
$$

In the perturbative calculation the investigated operators are sandwiched between offshell quark states. We shall denote the momentum of the incoming quark by $p$ and that of the outgoing quark by $p^{\prime}$. Our calculations are performed in Feynman gauge, the final numbers will be presented for the Wilson parameter $r=1$.

\section{Operators and mixing}

\subsection{Renormalisation and mixing in the one-loop approximation}

In this section we discuss renormalisation and mixing in general terms, on the lattice as well as in the continuum.

Let $\Gamma_{j}^{D}\left(p^{\prime}, p, \mu, g_{\mathrm{R}}, \epsilon\right)(j=1,2, \ldots, N)$ be the dimensionally regularised amputated vertex functions of $N$ mixing operators $\mathcal{O}_{j}$ of the same dimension calculated in $4-2 \epsilon$ 
dimensions. The corresponding Born terms are denoted by $\Gamma_{j}^{\text {Born }}\left(p^{\prime}, p\right)$. The operators potentially contributing to the mixing have to satisfy certain symmetry requirements. They should transform identically according to a given irreducible representation of $O(4)$ or $H(4)$, respectively, and they should have the same charge conjugation parity.

The (dimensionless) renormalised coupling constant $g_{\mathrm{R}}$ is related to the (dimensionful) bare coupling constant $g$ by

$$
g_{\mathrm{R}}^{2}=\mu^{-2 \epsilon} g^{2}\left(1+O\left(g^{2}\right)\right),
$$

where $\mu$ is the renormalisation scale. In one-loop perturbation theory we get results of the form

$$
\begin{aligned}
& \Gamma_{j}^{D}\left(p^{\prime}, p, \mu, g_{\mathrm{R}}, \epsilon\right)=\Gamma_{j}^{\text {Born }}\left(p^{\prime}, p\right) \\
& \quad+g_{\mathrm{R}}^{2}\left[\sum_{k=1}^{N} \gamma_{j k}^{V}\left(\frac{1}{\epsilon}-\gamma_{E}+\ln (4 \pi)-\ln \frac{\left(p^{\prime}+p\right)^{2}}{4 \mu^{2}}\right) \Gamma_{k}^{\mathrm{Born}}\left(p^{\prime}, p\right)+f_{j}\left(p^{\prime}, p\right)\right]+O\left(g_{\mathrm{R}}^{4}\right),
\end{aligned}
$$

where $\gamma_{E}=0.5772 \ldots$ is Euler's constant. As usual, contributions which vanish for $\epsilon \rightarrow 0$ have been omitted. In what follows, we systematically omit all contributions $O\left(g_{\mathrm{R}}^{4}\right)$. In the $\overline{\mathrm{MS}}$ scheme the renormalised vertex functions are then given by

$$
\begin{aligned}
& \Gamma_{j}^{R}\left(p^{\prime}, p, \mu, g_{\mathrm{R}}\right)=\Gamma_{j}^{\mathrm{Born}}\left(p^{\prime}, p\right) \\
& \quad+g_{\mathrm{R}}^{2}\left[\sum_{k=1}^{N} \gamma_{j k}^{V} \cdot(-1) \cdot \ln \frac{\left(p^{\prime}+p\right)^{2}}{4 \mu^{2}} \Gamma_{k}^{\mathrm{Born}}\left(p^{\prime}, p\right)+f_{j}\left(p^{\prime}, p\right)\right] .
\end{aligned}
$$

In the absence of mixing with lower-dimensional operators the vertex functions regularised on a lattice can be written as

$$
\begin{aligned}
& \Gamma_{j}^{L}\left(p^{\prime}, p, a, g_{\mathrm{R}}\right)=\Gamma_{j}^{\text {Born }}\left(p^{\prime}, p\right) \\
& \quad+g_{\mathrm{R}}^{2}\left[\sum_{k=1}^{N} \gamma_{j k}^{V} \cdot(-1) \cdot \ln \frac{a^{2}\left(p^{\prime}+p\right)^{2}}{4} \Gamma_{k}^{\text {Born }}\left(p^{\prime}, p\right)+f_{j}^{L}\left(p^{\prime}, p\right)\right]
\end{aligned}
$$

up to terms vanishing as $a \rightarrow 0$. There should be an $N \times N$ matrix $\zeta$ such that the relation between the bare lattice vertex functions and the $\overline{\mathrm{MS}}$ renormalised vertex functions can be written as

$$
\Gamma_{j}^{R}\left(p^{\prime}, p, \mu, g_{\mathrm{R}}\right)=\sum_{k=1}^{N}\left(\delta_{j k}+g_{\mathrm{R}}^{2} \zeta_{j k}\right) \Gamma_{k}^{L}\left(p^{\prime}, p, a, g_{\mathrm{R}}\right) .
$$

So we should have

$$
\begin{aligned}
& \Gamma_{j}^{R}\left(p^{\prime}, p, \mu, g_{\mathrm{R}}\right)=\Gamma_{j}^{\mathrm{Born}}\left(p^{\prime}, p\right) \\
& \quad+g_{\mathrm{R}}^{2}\left[\sum_{k=1}^{N}\left(\zeta_{j k}-\gamma_{j k}^{V} \ln \frac{a^{2}\left(p^{\prime}+p\right)^{2}}{4}\right) \Gamma_{k}^{\mathrm{Born}}\left(p^{\prime}, p\right)+f_{j}^{L}\left(p^{\prime}, p\right)\right] .
\end{aligned}
$$


Comparing with (8) we arrive at

$$
\sum_{k=1}^{N}\left(\zeta_{j k}-\gamma_{j k}^{V} \ln \left(a^{2} \mu^{2}\right)\right) \Gamma_{k}^{\text {Born }}\left(p^{\prime}, p\right)+f_{j}^{L}\left(p^{\prime}, p\right)-f_{j}\left(p^{\prime}, p\right)=0 .
$$

As this equation must hold for arbitrary momenta $p^{\prime}, p$, there should be constants $c_{j k}^{V}$ such that

$$
f_{j}^{L}\left(p^{\prime}, p\right)-f_{j}\left(p^{\prime}, p\right)=\sum_{k=1}^{N} c_{j k}^{V} \Gamma_{k}^{\text {Born }}\left(p^{\prime}, p\right) .
$$

This fixes the matrix $\zeta_{j k}$ :

$$
\zeta_{j k}=\gamma_{j k}^{V} \ln \left(a^{2} \mu^{2}\right)-c_{j k}^{V}
$$

If the coefficients $c_{j k}^{V}$ are uniquely determined, exactly all $N$ operators mix to one-loop accuracy. It might happen that certain operators contribute to mixing only in higher orders. In that case $N$ is decreased. If Eq. (13) cannot be satisfied, this shows that at least one mixing operator has been overlooked.

Mixing with lower-dimensional operators leads to the appearance of additional terms on the r.h.s. of Eq. (9). For example, consider the case of a single operator which mixes with $\mathcal{O}_{j}$ and whose dimension is one unit smaller. (Such a case will appear in our applications.) Then we get instead of (9)

$$
\begin{aligned}
& \Gamma_{j}^{L}\left(p^{\prime}, p, a, g_{\mathrm{R}}\right)=\Gamma_{j}^{\mathrm{Born}}\left(p^{\prime}, p\right) \\
& \quad+g_{\mathrm{R}}^{2}\left[\sum_{k=1}^{N} \gamma_{j k}^{V} \cdot(-1) \cdot \ln \frac{a^{2}\left(p^{\prime}+p\right)^{2}}{4} \Gamma_{k}^{\mathrm{Born}}\left(p^{\prime}, p\right)+\frac{1}{a} c_{j} \Gamma^{\mathrm{Born}}\left(p^{\prime}, p\right)+f_{j}^{L}\left(p^{\prime}, p\right)\right],
\end{aligned}
$$

where $\Gamma^{\text {Born }}\left(p^{\prime}, p\right)$ is the Born term of the additional lower-dimensional operator. The $1 / a$ contribution has to be subtracted from $\Gamma_{j}^{L}\left(p^{\prime}, p, a, g_{\mathrm{R}}\right)$ before the connection with $\Gamma_{j}^{R}\left(p^{\prime}, p, \mu, g_{\mathrm{R}}\right)$ can be established, i.e. in (10) $\Gamma_{k}^{L}\left(p^{\prime}, p, a, g_{\mathrm{R}}\right)$ has to be replaced by

$$
\Gamma_{k}^{L}\left(p^{\prime}, p, a, g_{\mathrm{R}}\right)-\frac{g_{\mathrm{R}}^{2}}{a} c_{k} \Gamma^{\mathrm{Born}}\left(p^{\prime}, p\right) .
$$

Then Eq. (13) is obtained as before.

In order to compute the matrix $Z_{j k}$ of renormalisation and mixing coefficients we note that the connection between the bare lattice vertex functions and the $\overline{\mathrm{MS}}$ renormalised vertex functions can be written as

$$
\Gamma_{j}^{R}\left(p^{\prime}, p, \mu, g_{\mathrm{R}}\right)=Z_{\psi}^{-1} \sum_{k=1}^{N} Z_{j k} \Gamma_{k}^{L}\left(p^{\prime}, p, a, g_{\mathrm{R}}\right)
$$


with the quark wave function renormalisation constant ${ }^{2} Z_{\psi}$. (In the presence of mixing with a lower-dimensional operator, $\Gamma_{k}^{L}\left(p^{\prime}, p, a, g_{\mathrm{R}}\right)$ has again to be replaced by the subtracted expression (16) $)$. Comparison with (10) yields

$$
Z_{\psi}^{-1} Z_{j k}=\delta_{j k}+g_{\mathrm{R}}^{2} \zeta_{j k}
$$

The quark wave function renormalisation constant is calculated from the quark propagator. Write the lattice regularised inverse quark propagator in Feynman gauge (after subtracting a linearly diverging contribution $\sim 1 / a$ ) as

$$
S_{L}^{-1}(p)=i \not p\left[1+\frac{g_{R}^{2} C_{F}}{16 \pi^{2}}\left(-\ln \left(a^{2} p^{2}\right)-\sigma_{L}\right)\right]
$$

where $\sigma_{L}=11.8524$ (see e.g. [16]) for Wilson fermions. In dimensional regularisation the inverse quark propagator is given by

$$
S_{D}^{-1}(p)=i \not p\left[1+\frac{g_{R}^{2} C_{F}}{16 \pi^{2}}\left(\frac{1}{\epsilon}-\gamma_{E}+\ln (4 \pi)-\ln \frac{p^{2}}{\mu^{2}}+1\right)\right]
$$

and we get for the $\overline{\mathrm{MS}}$ renormalised propagator

$$
S_{R}^{-1}(p)=i \not p\left[1+\frac{g_{R}^{2} C_{F}}{16 \pi^{2}}\left(-\ln \frac{p^{2}}{\mu^{2}}+1\right)\right] .
$$

Defining $Z_{\psi}$ such that

$$
S_{R}^{-1}(p)=Z_{\psi}^{-1} S_{L}^{-1}(p)
$$

we have

$$
Z_{\psi}=1-\frac{g_{R}^{2} C_{F}}{16 \pi^{2}}\left(\ln \left(a^{2} \mu^{2}\right)+1+\sigma_{L}\right)
$$

With the help of this result we get from (14) and (18) for the matrix of the renormalisation and mixing coefficients

$$
Z_{j k}=\delta_{j k}+g_{\mathrm{R}}^{2}\left[\left(\gamma_{j k}^{V}-\delta_{j k} \frac{C_{F}}{16 \pi^{2}}\right) \ln \left(a^{2} \mu^{2}\right)-c_{j k}^{V}-\delta_{j k} \frac{C_{F}}{16 \pi^{2}}\left(1+\sigma_{L}\right)\right] .
$$

The basic computational task is thus to find the functions $f_{j}\left(p^{\prime}, p\right)$ and $f_{j}^{L}\left(p^{\prime}, p\right)$ in Eqs. (7) and (9) (or rather their difference). Then we can compute the coefficients $c_{j k}^{V}$ from Eq. (13) and use Eq. (24) to get the desired renormalisation and mixing coefficients. If we are only interested in the renormalisation of one particular operator, corresponding to $j=1$ say, it is sufficient to restrict the calculations to the case $j=1$.

\footnotetext{
${ }^{2}$ Often the quark wave function renormalisation constant is defined as $1 / Z_{\psi}$.
} 


\subsection{Contributing operators for special representations}

Let us introduce the self-explaining notations for operators with covariant and external ordinary derivatives

$$
\begin{aligned}
\mathcal{O}_{\mu \nu \omega}^{D D} & =-\frac{1}{4} \bar{\psi} \gamma_{\mu} \stackrel{\leftrightarrow}{D}_{\nu} \stackrel{\leftrightarrow}{D}_{\omega} \psi, \\
\mathcal{O}_{\mu \nu \omega}^{\partial D} & =-\frac{1}{4} \partial_{\nu}\left(\bar{\psi} \gamma_{\mu} \stackrel{\leftrightarrow}{D}_{\omega} \psi\right) \\
\mathcal{O}_{\mu \nu \omega}^{\partial \partial} & =-\frac{1}{4} \partial_{\nu} \partial_{\omega}\left(\bar{\psi} \gamma_{\mu} \psi\right)
\end{aligned}
$$

and

$$
\begin{aligned}
\mathcal{O}_{\mu \nu \omega}^{5, D D} & =-\frac{1}{4} \bar{\psi} \gamma_{\mu} \gamma_{5} \stackrel{\leftrightarrow}{D}_{\nu} \stackrel{\leftrightarrow}{D}_{\omega} \psi \\
\mathcal{O}_{\mu \nu \omega}^{5, \partial D} & =-\frac{1}{4} \partial_{\nu}\left(\bar{\psi} \gamma_{\mu} \gamma_{5} \stackrel{\leftrightarrow}{D}_{\omega} \psi\right) \\
\mathcal{O}_{\mu \nu \omega}^{5, \partial \partial} & =-\frac{1}{4} \partial_{\nu} \partial_{\omega}\left(\bar{\psi} \gamma_{\mu} \gamma_{5} \psi\right)
\end{aligned}
$$

as well as the lower-dimensional operators

$$
\mathcal{O}_{\mu \nu \omega}^{D}=-\frac{i}{2} \bar{\psi}\left[\gamma_{\mu}, \gamma_{\nu}\right] \stackrel{\leftrightarrow}{D}_{\omega} \psi, \quad \mathcal{O}_{\mu \nu \omega}^{\partial}=-\frac{i}{2} \partial_{\omega}\left(\bar{\psi}\left[\gamma_{\mu}, \gamma_{\nu}\right] \psi\right)
$$

For completeness we include here also operators contributing to non-forward transversity matrix elements with two derivatives which were not considered in Eqs. (2) and (4):

$$
\mathcal{O}_{\mu \nu \omega \sigma}^{T, D D}=-\frac{1}{4} \bar{\psi}\left[\gamma_{\mu}, \gamma_{\nu}\right] \stackrel{\leftrightarrow}{D}_{\omega} \stackrel{\leftrightarrow}{D}_{\sigma} \psi, \quad \mathcal{O}_{\mu \nu \omega \sigma}^{T, \partial \partial}=-\frac{1}{4} \partial_{\omega} \partial_{\sigma}\left(\bar{\psi}\left[\gamma_{\mu}, \gamma_{\nu}\right] \psi\right)
$$

As short-hand notations we use in the following (cf. [12])

$$
\begin{aligned}
\mathcal{O}_{\left\{\nu_{1} \nu_{2} \nu_{3}\right\}} & =\frac{1}{6}\left(\mathcal{O}_{\nu_{1} \nu_{2} \nu_{3}}+\mathcal{O}_{\nu_{1} \nu_{3} \nu_{2}}+\mathcal{O}_{\nu_{2} \nu_{1} \nu_{3}}+\mathcal{O}_{\nu_{2} \nu_{3} \nu_{1}}+\mathcal{O}_{\nu_{3} \nu_{1} \nu_{2}}+\mathcal{O}_{\nu_{3} \nu_{2} \nu_{1}}\right) \\
\mathcal{O}_{\left\|\nu_{1} \nu_{2} \nu_{3}\right\|} & =\mathcal{O}_{\nu_{1} \nu_{2} \nu_{3}}-\mathcal{O}_{\nu_{1} \nu_{3} \nu_{2}}+\mathcal{O}_{\nu_{3} \nu_{1} \nu_{2}}-\mathcal{O}_{\nu_{3} \nu_{2} \nu_{1}}-2 \mathcal{O}_{\nu_{2} \nu_{3} \nu_{1}}+2 \mathcal{O}_{\nu_{2} \nu_{1} \nu_{3}} \\
\mathcal{O}_{\left\langle\left\langle\nu_{1} \nu_{2} \nu_{3}\right\rangle\right\rangle} & =\mathcal{O}_{\nu_{1} \nu_{2} \nu_{3}}+\mathcal{O}_{\nu_{1} \nu_{3} \nu_{2}}-\mathcal{O}_{\nu_{3} \nu_{1} \nu_{2}}-\mathcal{O}_{\nu_{3} \nu_{2} \nu_{1}}
\end{aligned}
$$

First we consider the operator

$$
\mathcal{O}_{\{124\}}^{D D}=-\frac{1}{4} \bar{\psi} \gamma_{\{1} \stackrel{\leftrightarrow}{D}_{2} \stackrel{\leftrightarrow}{D}_{4\}} \psi
$$


Its charge conjugation parity is $C=-1$ and it is a member of an irreducible multiplet of operators transforming according to the representation $\tau_{2}^{(4)}$ of $H(4)$ [12. Here $\tau_{k}^{(l)}$ denotes an irreducible representation of $H(4)$ with dimension $l$, and $k=1,2, \ldots$ labels inequivalent representations of the same dimension. This operator can only mix with

$$
\mathcal{O}_{\{124\}}^{\partial \partial}=-\frac{1}{4} \partial_{\{2} \partial_{4}\left(\bar{\psi} \gamma_{1\}} \psi\right)
$$

Next we examine the operator

$$
\mathcal{O}_{1}=\mathcal{O}_{\{114\}}^{D D}-\frac{1}{2}\left(\mathcal{O}_{\{224\}}^{D D}+\mathcal{O}_{\{334\}}^{D D}\right)
$$

It has already been used in lattice computations of forward hadronic matrix elements, because in this case it suffers only from rather mild mixing problems. It belongs to the representation $\tau_{1}^{(8)}$ with $C=-1$.

Taking into account also external ordinary derivatives, one finds the following operators which transform identically and could therefore mix with $(34)^{3}$ :

$$
\begin{aligned}
\mathcal{O}_{2} & =\mathcal{O}_{\{114\}}^{\partial \partial}-\frac{1}{2}\left(\mathcal{O}_{\{224\}}^{\partial \partial}+\mathcal{O}_{\{334\}}^{\partial \partial}\right), \\
\mathcal{O}_{3} & =\mathcal{O}_{\langle\langle 114\rangle\rangle}^{D D}-\frac{1}{2}\left(\mathcal{O}_{\langle\langle 224\rangle\rangle}^{D D}+\mathcal{O}_{\langle\langle 334\rangle\rangle}^{D D}\right), \\
\mathcal{O}_{4} & =\mathcal{O}_{\langle\langle 114\rangle\rangle}^{\partial \partial}-\frac{1}{2}\left(\mathcal{O}_{\langle\langle 224\rangle\rangle}^{\partial \partial}+\mathcal{O}_{\langle\langle 334\rangle\rangle}^{\partial \partial}\right), \\
\mathcal{O}_{5} & =\mathcal{O}_{\|\mid 213\|}^{5, \partial D}, \\
\mathcal{O}_{6} & =\mathcal{O}_{\langle\langle 213\rangle\rangle}^{5, \partial D}, \\
\mathcal{O}_{7} & =\mathcal{O}_{\| \mid D D}^{5, D D}
\end{aligned}
$$

and the lower-dimensional operator

$$
\mathcal{O}_{8}=\mathcal{O}_{411}^{\partial}-\frac{1}{2}\left(\mathcal{O}_{422}^{\partial}+\mathcal{O}_{433}^{\partial}\right)
$$

As an "axial" analogue of (32) we consider the operator $\mathcal{O}_{\{124\}}^{5, D D}$

$$
\mathcal{O}_{\{124\}}^{5, D D}=-\frac{1}{4} \bar{\psi} \gamma_{\{1} \stackrel{\leftrightarrow}{D}_{2} \stackrel{\leftrightarrow}{D}_{4\}} \gamma_{5} \psi
$$

\footnotetext{
${ }^{3}$ The charge conjugation parities $C$ of $\mathcal{O}_{7}$ and $\mathcal{O}_{5}$ coincide since $\mathcal{O}_{7}$ is antisymmetric in the indices of the covariant derivatives.
} 
which can mix with

$$
\mathcal{O}_{\{124\}}^{5, \partial \partial}=-\frac{1}{4} \partial_{\{2} \partial_{4}\left(\bar{\psi} \gamma_{1\}} \gamma_{5} \psi\right)
$$

(37) and (38) belong to $\tau_{3}^{(4)}$ with $C=+1$.

Similarly, we have as a counterpart of (34) the operator

$$
\mathcal{O}_{1}^{5}=\mathcal{O}_{\{114\}}^{5, D D}-\frac{1}{2}\left(\mathcal{O}_{\{224\}}^{5, D D}+\mathcal{O}_{\{334\}}^{5, D D}\right)
$$

Its charge conjugation parity is $C=+1$ and it is a member of an irreducible multiplet of operators transforming according the representation $\tau_{2}^{(8)}$ of $H(4)$ [12].

The following operators with identical transformation behaviour could potentially mix with (39):

$$
\begin{aligned}
\mathcal{O}_{2}^{5} & =\mathcal{O}_{\{114\}}^{5, \partial \partial}-\frac{1}{2}\left(\mathcal{O}_{\{224\}}^{5, \partial \partial}+\mathcal{O}_{\{334\}}^{5, \partial \partial}\right), \\
\mathcal{O}_{3}^{5} & =\mathcal{O}_{\langle\langle 114\rangle\rangle}^{5, D D}-\frac{1}{2}\left(\mathcal{O}_{\langle\langle 224\rangle\rangle}^{5, D D}+\mathcal{O}_{\langle\langle 334\rangle\rangle}^{5, D D}\right), \\
\mathcal{O}_{4}^{5} & =\mathcal{O}_{\langle\langle 114\rangle\rangle}^{5, \partial \partial}-\frac{1}{2}\left(\mathcal{O}_{\langle\langle 224\rangle\rangle}^{5, \partial \partial}+\mathcal{O}_{\langle\langle 334\rangle\rangle}^{5, \partial \partial}\right), \\
\mathcal{O}_{5}^{5} & =\mathcal{O}_{\|\mid 213\|}^{\partial D}, \\
\mathcal{O}_{6}^{5} & =\mathcal{O}_{\langle\langle 213\rangle\rangle}^{\partial D}, \\
\mathcal{O}_{7}^{5} & =\mathcal{O}_{|| 213 \|}^{D D}
\end{aligned}
$$

and the lower-dimensional operator

$$
\mathcal{O}_{8}^{5}=\mathcal{O}_{123}^{D}-2 \mathcal{O}_{231}^{D}-\mathcal{O}_{132}^{D}
$$

For the operators (28) we consider the representations $\tau_{2}^{(3)}, \tau_{3}^{(3)}$ and $\tau_{2}^{(6)}$ with $C=$ -1 [13]. In the case of $\tau_{2}^{(3)}$ we choose the representative operator

$$
\mathcal{O}_{1}^{T}=\mathcal{O}_{4\{123\}}^{T, D D}
$$

which may mix with

$$
\mathcal{O}_{2}^{T}=\mathcal{O}_{4\{123\}}^{T, \partial \partial}
$$

For $\tau_{3}^{(3)}$ we take

$$
\mathcal{O}_{3}^{T}=-\mathcal{O}_{1\{133\}}^{T, D D}+\mathcal{O}_{1\{144\}}^{T, D D}-\mathcal{O}_{2\{233\}}^{T, D D}+\mathcal{O}_{2\{244\}}^{T, D D}-2 \mathcal{O}_{3\{344\}}^{T, D D}
$$


mixing with

$$
\mathcal{O}_{4}^{T}=-\mathcal{O}_{1\{133\}}^{T, \partial \partial}+\mathcal{O}_{1\{144\}}^{T, \partial \partial}-\mathcal{O}_{2\{233\}}^{T, \partial \partial}+\mathcal{O}_{2\{244\}}^{T, \partial \partial}-2 \mathcal{O}_{3\{344\}}^{T, \partial \partial} .
$$

The operator

$$
\mathcal{O}_{5}^{T}=\mathcal{O}_{13\{32\}}^{T, D D}+\mathcal{O}_{23\{31\}}^{T, D D}-\mathcal{O}_{14\{42\}}^{T, D D}-\mathcal{O}_{24\{41\}}^{T, D D}
$$

belonging to the representation $\tau_{2}^{(6)}$ also mixes with only one additional operator:

$$
\mathcal{O}_{6}^{T}=\mathcal{O}_{13\{32\}}^{T, \partial \partial}+\mathcal{O}_{23\{31\}}^{T, \partial \partial}-\mathcal{O}_{14\{42\}}^{T, \partial \partial}-\mathcal{O}_{24\{41\}}^{T, \partial \partial} .
$$

Other representations show a more complicated mixing behaviour and will be not considered here.

\section{One-loop calculation of non-forward matrix ele- ments}

\subsection{Calculational technique}

We calculate the matrix elements of the operators in one-loop lattice perturbation theory in the infinite volume limit following Kawai et al. 14. The computation is performed symbolically adopting and significantly extending a Mathematica program package developed originally for the case of moments of structure functions using Wilson [15], clover [16] and overlap fermions [17].

Using that approach we have full analytic control over pole cancellation. The Lorentz index structure of the matrix elements is left completely free, so that we are able to construct all representations of the hypercubic group for the second moments in the nonforward case. The analytic derivation is disconnected from the numerical calculation of finite lattice integrals. These integrals are calculated to a high accuracy and are given as look-up tables.

To be more precise, we recapitulate in short the strategy [14, 15] for calculating the diagrams adapted to our case of two distinct external momenta $p \neq p^{\prime}$. Basically we have to evaluate a typical lattice integral of the form

$$
\mathcal{I}_{\mu_{1} \cdots \mu_{n}}\left(a, p^{\prime}, p\right)=\int_{-\pi / a}^{\pi / a} \frac{d^{4} k}{(2 \pi)^{4}} \mathcal{K}_{\mu_{1} \cdots \mu_{n}}\left(a, p^{\prime}, p, k\right)
$$


where the integration is performed over the first Brillouin zone. The numerator of the kernel $\mathcal{K}_{\mu_{1} \cdots \mu_{n}}\left(a, p^{\prime}, p, k\right)$ is a polynomial in sines and cosines of the lattice momenta $k$, the denominator contains the denominators of lattice quark and gluon propagators. Such an integral $\mathcal{I}$ is calculated by splitting it into two parts

$$
\mathcal{I}=\tilde{\mathcal{I}}+(\mathcal{I}-\tilde{\mathcal{I}}) .
$$

Here $\tilde{\mathcal{I}}$ denotes the Taylor expansion of the original integral $\mathcal{I}$ in the external momenta

$$
\begin{aligned}
\tilde{\mathcal{I}}\left(a, p^{\prime}, p\right) & =\mathcal{I}(a, 0,0) \\
& +\sum_{\alpha}\left\{\left.p_{\alpha}^{\prime} \frac{\partial \mathcal{I}\left(a, p^{\prime}, p\right)}{\partial p_{\alpha}^{\prime}}\right|_{p^{\prime}=p=0}+\left.p_{\alpha} \frac{\partial \mathcal{I}\left(a, p^{\prime}, p\right)}{\partial p_{\alpha}}\right|_{p^{\prime}=p=0}\right\}+\ldots
\end{aligned}
$$

where the order of the expansion is given by the degree of ultraviolet (UV) divergence of $\mathcal{I}$. As a consequence, the difference $\mathcal{I}-\tilde{\mathcal{I}}$ is $U V$ finite and can be calculated in the (Euclidean) continuum, taking the limit $a \rightarrow 0$. The original UV poles appear now as infrared (IR) poles in the Taylor expansion and are regularised using dimensional regularisation with $d>4$. Since in that case $\left.\tilde{\mathcal{I}}\right|_{a \rightarrow 0}$ vanishes, we have $\left.(\mathcal{I}-\tilde{\mathcal{I}})\right|_{a \rightarrow 0}=\left.\mathcal{I}\right|_{a \rightarrow 0} \equiv \mathcal{I}^{\text {cont }}\left(p^{\prime}, p\right)$ and the second contribution to the final lattice result is just a one-loop continuum calculation in dimensional regularisation.

The first (Taylor expanded) part is calculated in $d=4-2 \epsilon$ dimensions at finite $a$, the poles in $\epsilon$ analytically cancel those of the second part $\mathcal{I}^{\text {cont }}$. Note that the first part depends on the external momenta only via the Taylor expansion, therefore the lattice integrals (the expansion coefficients) are just numbers (independent of $p$ and $p^{\prime}$ ).

In the Euclidean continuum part $\mathcal{I}^{\text {cont }}\left(p^{\prime}, p\right)$ the finite contribution depends on the (different) external momenta in a complicated manner. As in Minkowski space, the finite part of three-point functions with three different propagators is difficult to represent in a simple compact analytic form (see, e.g., [18]). In parametrising that contribution we have chosen the following form

$$
\mathcal{I}_{\mu_{1} \cdots \mu_{n}}^{\mathrm{cont}}\left(p^{\prime}, p\right)=\frac{1}{\epsilon} A_{\mu_{1} \cdots \mu_{n}}\left(p^{\prime}, p\right)+\sum_{i, j, k, m} B_{\mu_{1} \cdots \mu_{n}}\left(p^{\prime}, p, i, j, k, m\right) \mathcal{F}\left(i, j, k, m, p^{\prime}, p\right) .
$$

In (51) the functions $A_{\mu_{1} \cdots \mu_{n}}\left(p^{\prime}, p\right)$ (known analytically) and $B_{\mu_{1} \cdots \mu_{n}}\left(p^{\prime}, p, i, j, k, m\right)$ contain the general index structure assigned to the external momenta $p$ and $p^{\prime}$. The symbols $\mathcal{F}$ stand for the remaining integrals (finite for $p \neq p^{\prime}$ ) over the Feynman parameters ${ }^{4}$ :

$$
\mathcal{F}\left(i, j, k, m, p^{\prime}, p\right)=\int_{0}^{1} d x \int_{0}^{1-x} d y x^{i} y^{j}\left(Q^{2}\left(x, y, p^{\prime}, p\right)\right)^{k} \ln ^{m} \frac{Q^{2}\left(x, y, p^{\prime}, p\right)}{\mu^{2}}
$$

\footnotetext{
${ }^{4}$ One Feynman parameter integration can be performed analytically, leading, however, to lengthy expressions for the individual integrals. We have checked that for our continuum results we recover the known results of the forward case.
} 
with

$$
Q^{2}\left(x, y, p^{\prime}, p\right)=p^{2} x(1-x)+p^{2} y(1-y)-2 p \cdot p^{\prime} x y .
$$

Only few combinations of the integers $i, j, k, m$ are actually needed in the "continuum part" of the one-loop calculation. But compact expressions for the $\mathcal{F}\left(i, j, k, m, p^{\prime}, p\right)$ do not seem to exist.

As we have shown in Section 2.1. in order to calculate the finite renormalisation matrix converting the lattice result to the $\overline{\mathrm{MS}}$ scheme we have to find the difference between the lattice and the continuum one-loop contribution $f_{j}^{L}\left(p^{\prime}, p\right)-f_{j}\left(p^{\prime}, p\right)$, and similarly for the wave function renormalisation. Therefore, we do not need the explicit form of the finite continuum one-loop contribution (which is essentially given by the $\mathcal{F}$ 's), since it cancels exactly the part coming from the second term of (49). As a consequence, we do not present $\mathcal{F}$-integrals here. However, choosing other schemes, the finite contributions to $\mathcal{I}_{\mu_{1} \cdots \mu_{n}}\left(p^{\prime}, p\right)^{\text {cont }}$ might be needed.

\subsection{One-loop renormalisation matrices for the chosen represen- tations}

To calculate the one-loop contributions for the above operators between quark states we have to take into account self energy and amputated Green function diagrams. The quark self energy diagrams (contributing to the quark wave function renormalisation) are shown in Fig. 1] Denoting the operator insertions by a black dot, the vertex and tadpole

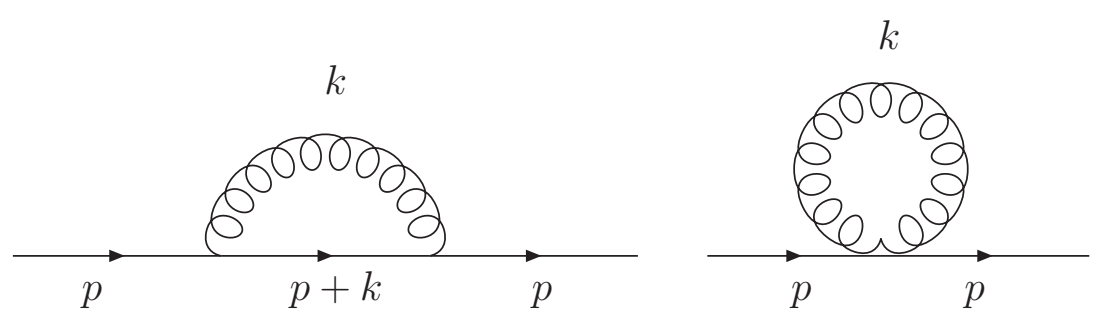

Figure 1: One-loop diagrams contributing to the quark self-energy.

diagrams (Fig. 2) as well as the "cockscomb" diagrams (Fig. 3) contribute in one-loop order to the amputated Green functions.

We present the results for the $\mathrm{Z}$ factors of the operators discussed in Section 2.2 in 

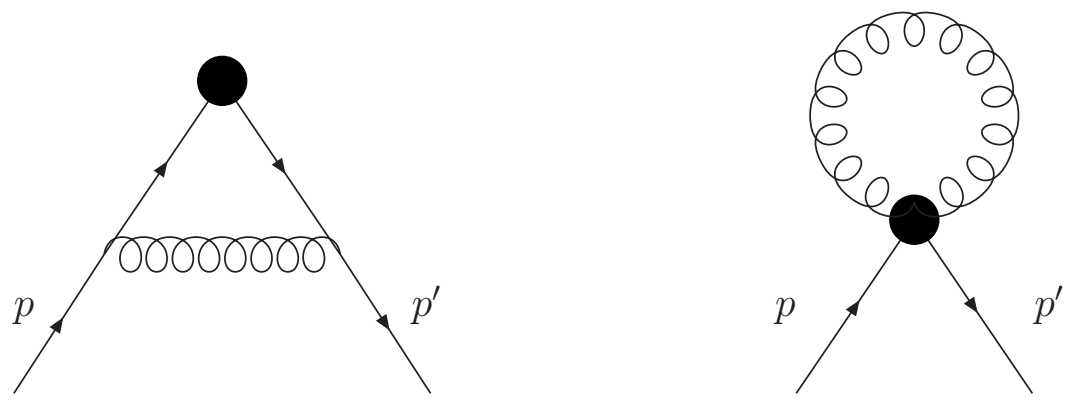

Figure 2: One-loop vertex and tadpole diagrams.
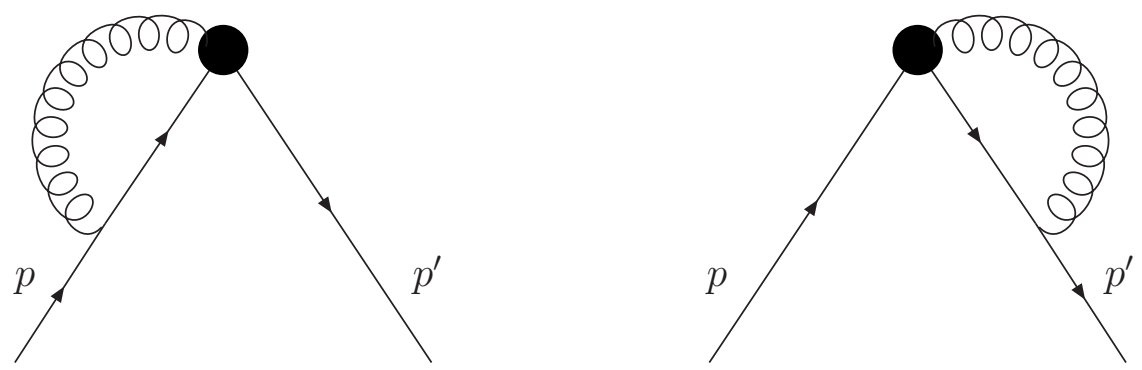

Figure 3: One-loop cockscomb diagrams.

the generic form

$$
Z_{j k}^{(m)}=\delta_{j k}-\frac{g_{R}^{2} C_{F}}{16 \pi^{2}}\left(\gamma_{j k} \ln \left(a^{2} \mu^{2}\right)+c_{j k}^{(m)}\right)
$$

where (cf. (24))

$$
\gamma_{j k}=\delta_{j k}-\frac{16 \pi^{2}}{C_{F}} \gamma_{j k}^{V}, \quad c_{j k}^{(m)}=\delta_{j k}\left(1+\sigma_{L}\right)+\frac{16 \pi^{2}}{C_{F}} c_{j k}^{V}
$$

The superscript $(m)$ with $m=I, I I$ distinguishes the realisations I and II of the covariant derivatives, which are explained in the Appendix. 
3.2.1 $\mathcal{O}_{\{124\}}^{D D}\left(\tau_{2}^{(4)}, C=-1\right)$

For this representation we find mixing between $\mathcal{O}_{\{124\}}^{D D}$ (32) and $\mathcal{O}_{\{124\}}^{\partial \partial}$ (33). The corresponding $2 \times 2$-mixing matrices are

$$
\begin{gathered}
\gamma_{j k}=\left(\begin{array}{rr}
\frac{25}{6} & -\frac{5}{6} \\
0 & 0
\end{array}\right), \\
c_{j k}^{(I, I I)}=\left(\begin{array}{rr}
-11.5632 & 0.0241 \\
0 & 20.6178
\end{array}\right) .
\end{gathered}
$$

The anomalous dimension matrix was calculated earlier [11]. The element $c_{11}=-11.5632$ in the matrix (56) is known from our previous calculation in the forward case [15] and $c_{22}$ comes from the renormalisation of the local vector current [16]. The matrix $c_{j k}^{(I, I I)}$ shows a very small mixing between the operators $\mathcal{O}_{\{124\}}^{D D}$ and $\mathcal{O}_{\{124\}}^{\partial \partial}$. Thus it may be justified to neglect the mixing in practical applications.

\subsection{2 $\mathcal{O}_{1}\left(\tau_{1}^{(8)}, C=-1\right)$}

We consider the mixing of operators having the same dimension first. The relevant operators have been defined in Section 2.2. To one-loop accuracy the operator $\mathcal{O}_{7}$ in Eq. (35) does not contribute and we have to consider the following mixing set:

$$
\left\{\mathcal{O}_{1}, \mathcal{O}_{2}, \mathcal{O}_{3}, \mathcal{O}_{4}, \mathcal{O}_{5}, \mathcal{O}_{6}\right\}
$$

The anomalous dimension matrix is

$$
\gamma_{j k}=\left(\begin{array}{rrrrrr}
\frac{25}{6} & -\frac{5}{6} & 0 & 0 & 0 & 0 \\
0 & 0 & 0 & 0 & 0 & 0 \\
0 & 0 & \frac{7}{6} & -\frac{5}{6} & 1 & -\frac{3}{2} \\
0 & 0 & 0 & 0 & 0 & 0 \\
0 & 0 & 0 & 0 & 2 & -2 \\
0 & 0 & 0 & 0 & -\frac{2}{3} & \frac{2}{3}
\end{array}\right)
$$

and the finite part of the mixing matrix is given by

$$
c_{j k}^{(I)}=\left(\begin{array}{rrrrrr}
-12.1274 & -2.7367 & 0.3685 & 0.9934 & 0.0156 & 0.1498 \\
0 & 20.6178 & 0 & 0 & 0 & 0 \\
3.3060 & 18.1841 & -14.8516 & -4.3023 & -0.9285 & 0.7380 \\
0 & 0 & 0 & 20.6178 & 0 & 0 \\
0 & 3.2644 & 0 & 0 & 0.3501 & 0.0149 \\
0 & 3.2644 & 0 & 0 & 0.0050 & 0.3600
\end{array}\right)
$$


or

$$
c_{j k}^{(I I)}=\left(\begin{array}{rrrrrr}
-12.1274 & 1.4913 & 0.3685 & -0.4160 & 0.0156 & 0.1498 \\
0 & 20.6178 & 0 & 0 & 0 & 0 \\
3.3060 & -8.01456 & -14.8516 & 4.3023 & -0.9285 & 0.7380 \\
0 & 0 & 0 & 20.6178 & 0 & 0 \\
0 & 3.2644 & 0 & 0 & 0.3501 & 0.0149 \\
0 & 3.2644 & 0 & 0 & 0.0050 & 0.3600
\end{array}\right)
$$

The matrices $c_{j k}^{(I, I I)}$ show a sizeable mixing of the operator $\mathcal{O}_{1}$ with other operators, especially $\mathcal{O}_{2}$ containing two external ordinary derivatives. This mixing becomes irrelevant in the forward case, where the matrix element of $\mathcal{O}_{2}$ vanishes. On the other hand, the mixing between the operators $\mathcal{O}_{1}$ and $\mathcal{O}_{3}$ is already known from our previous calculations in the forward case [15].

There is also a possible mixing between $\mathcal{O}_{1}$ and the lower-dimensional operator $\mathcal{O}_{8}$ ([36). Indeed, we find in the one-loop approximation that the vertex function of $\mathcal{O}_{1}$ contains a term $\propto 1 / a:$

$$
\left.\mathcal{O}_{1}\right|_{\frac{1}{a}-\text { part }}=\frac{g_{R}^{2} C_{F}}{16 \pi^{2}}(-0.5177) \frac{1}{a} \mathcal{O}_{8}^{\text {Born }} .
$$

This mixing leads to a contribution which diverges like the inverse lattice spacing in the continuum limit. The operator $\mathcal{O}_{8}$ has to be subtracted non-perturbatively from the operator $\mathcal{O}_{1}$ which might be a difficult task in simulations.

\subsection{3 $\mathcal{O}_{\{124\}}^{5, D D}\left(\tau_{3}^{(4)}, C=+1\right)$}

For this representation we find mixing between $\mathcal{O}_{\{124\}}^{5, D D}$ and $\mathcal{O}_{\{124\}}^{5, \partial \partial}$. The anomalous dimension matrix is given by (55), the finite contributions are collected in the matrix

$$
c_{j k}^{(I, I I)}=\left(\begin{array}{rr}
-12.1171 & 0.1667 \\
0 & 15.7963
\end{array}\right) .
$$

As in the case of the operator $\mathcal{O}_{\{124\}}^{D D}$ above, the mixing is rather small, and the diagonal matrix elements $c_{11}$ and $c_{22}$ can be inferred from previous work [15]. 


\subsection{4 $\mathcal{O}_{1}^{5}\left(\tau_{2}^{(8)}, C=+1\right)$}

First we discuss the mixing of operators of the same dimension. The set of contributing operators is found to be

$$
\left\{\mathcal{O}_{1}^{5}, \mathcal{O}_{2}^{5}, \mathcal{O}_{3}^{5}, \mathcal{O}_{4}^{5}, \mathcal{O}_{5}^{5}, \mathcal{O}_{6}^{5}\right\}
$$

As in the case of the operator $\mathcal{O}_{1}$, one operator - here $\mathcal{O}_{7}^{5}$ - does not contribute to mixing in one-loop order. The finite contributions for the two lattice derivatives are

$$
c_{j k}^{(I)}=\left(\begin{array}{rrrrrr}
-12.8609 & -2.0653 & 0.3490 & 0.8538 & 0.0511 & 0.0594 \\
0 & 15.7963 & 0 & 0 & 0 & 0 \\
3.4220 & 15.8207 & -15.3592 & -5.1639 & 0.1701 & -0.9431 \\
0 & 0 & 0 & 15.7963 & 0 & 0 \\
0 & -8.9124 & 0 & 0 & 0.9597 & -0.9597 \\
0 & -8.9124 & 0 & 0 & -0.3199 & 0.3199
\end{array}\right)
$$

and

$$
c_{j k}^{(I I)}=\left(\begin{array}{rrrrrr}
-12.8609 & 1.4894 & 0.3490 & -0.3311 & 0.0511 & 0.0594 \\
0 & 15.7963 & 0 & 0 & 0 & 0 \\
3.4220 & -7.3002 & -15.3592 & 2.5431 & 0.1701 & -0.9431 \\
0 & 0 & 0 & 15.7963 & 0 & 0 \\
0 & -8.9124 & 0 & 0 & 0.9597 & -0.9597 \\
0 & -8.9124 & 0 & 0 & -0.3199 & 0.3199
\end{array}\right) .
$$

The anomalous dimension matrix is the same as for the operators without $\gamma_{5}$, see (57). Again, some of the mixing coefficients may be non-negligible. The mixing between $\mathcal{O}_{1}^{5}$ and $\mathcal{O}_{3}^{5}$ is visible even in the forward case.

We also find mixing with a lower-dimensional operator, in this case it is the operator $\mathcal{O}_{8}^{5}$ (41). The corresponding contribution in the vertex function of $\mathcal{O}_{1}^{5}$ reads

$$
\left.\mathcal{O}_{1}^{5}\right|_{\frac{1}{a}-\text { part }}=\frac{g_{R}^{2} C_{F}}{16 \pi^{2}}(-0.2523) \frac{1}{a} \mathcal{O}_{8}^{5, \text { Born }} .
$$

\subsection{5 $\mathcal{O}_{1}^{T}\left(\tau_{2}^{(3)}, C=-1\right)$}

For the mixing between the operators $\mathcal{O}_{1}^{T}(42)$ and $\mathcal{O}_{2}^{T}$ (43) the matrix of anomalous dimensions is given by

$$
\gamma_{j k}=\left(\begin{array}{rr}
\frac{13}{3} & -\frac{2}{3} \\
0 & 1
\end{array}\right)
$$


and the finite contributions are

$$
c_{j k}^{(I, I I)}=\left(\begin{array}{rr}
-11.5483 & 0.2189 \\
0 & 17.0181
\end{array}\right) .
$$

\subsection{6 $\mathcal{O}_{3}^{T}\left(\tau_{3}^{(3)}, C=-1\right)$}

The mixing between the operators $\mathcal{O}_{3}^{T}$ (44) and $\mathcal{O}_{4}^{T}$ (45) leads to the same anomalous dimension matrix (65) as the previous case. For the finite pieces we obtain

$$
c_{j k}^{(I, I I)}=\left(\begin{array}{rr}
-11.8688 & 0.2753 \\
0 & 17.0181
\end{array}\right) .
$$

\subsection{7 $\mathcal{O}_{5}^{T}\left(\tau_{2}^{(6)}, C=-1\right)$}

For the operators $\mathcal{O}_{5}^{T}$ (46) and $\mathcal{O}_{6}^{T}$ (47) we find the finite mixing contributions

$$
c_{j k}^{(I, I I)}=\left(\begin{array}{rr}
-11.7477 & 0.2380 \\
0 & 17.0181
\end{array}\right)
$$

with the identical anomalous dimension matrix (65).

\section{Summary}

Within the framework of lattice QCD with Wilson fermions and Wilson's plaquette action for the gauge fields we have calculated the one-loop quark matrix elements of operators needed for the second moments of GPDs and meson distribution amplitudes. From these we have determined the matrices of renormalisation and mixing coefficients in the $\overline{\mathrm{MS}}$ scheme. The operators contributing to the considered moments in the continuum contain two derivatives and are hence of dimension 5. On the lattice we should take operators with definite C-parity belonging to multiplets which transform irreducibly under the hypercubic group. We have selected some operators commonly used in numerical simulations of ordinary parton distributions. While these distributions are extracted from forward matrix elements, we have to consider non-forward matrix elements in order to study GPDs. This circumstance enlarges the set of potentially mixing operators, because also operators with external ordinary derivatives have to be taken into account. 
For the representations $\left(\tau_{2}^{(4)}, C=-1\right)$ and $\left(\tau_{3}^{(4)}, C=+1\right)$ there are only two multiplets of operators of dimension $\leq 5$. Actually, they are all of dimension 5, and the mixing pattern is the same as in the continuum. The mixing coefficients turn out to be quite small.

In the case of the representations $\left(\tau_{1}^{(8)}, C=-1\right)$ and $\left(\tau_{2}^{(8)}, C=+1\right)$ we have eight multiplets of operators of dimension $\leq 5$. In the one-loop approximation one of them cannot yet contribute, but the remaining seven multiplets do actually show up. Six of them are of dimension 5, but there is also one multiplet of operators of dimension 4 . For these representations some of the mixing coefficients are larger than in the above cases. In particular the mixing with the lower-dimensional operators, which will probably receive sizeable non-perturbative corrections, might lead to difficulties in the numerical simulations: 1/a effects are hard to get under control.

In the operators just mentioned the Dirac matrix is either $\gamma_{\mu}$ or $\gamma_{\mu} \gamma_{5}$. In connection with (generalised) transversity distributions operators with $\left[\gamma_{\mu}, \gamma_{\nu}\right]$ are of interest. Here we restricted ourselves to cases where only mixing with the external derivative counterparts is possible and found rather small mixing coefficients.

The results presented in this paper were obtained for Wilson fermions. However, numerical simulations will be performed with clover or overlap fermions. Therefore, the numbers given in the preceding section can only serve as first hints at the problems which will occur in these calculations, especially the mixing problem. The sets of mixing operators, on the other hand, follow from symmetry arguments and are therefore universally valid, although additional symmetries may lead to further restrictions, e.g. in the case of overlap fermions. It should also be noted that the overall normalisations of the operators are somewhat arbitrary. So some care has to be exercised when using our results in concrete applications.

\section{Acknowledgements}

We thank O. V. Tarasov for useful discussions. This work is supported by DFG under contract FOR 465 (Forschergruppe Gitter-Hadronen-Phänomenologie). 


\section{Appendix: Feynman rules}

We need Feynman rules for the lattice operators (25), (26) and (28). Denoting the momentum of the outgoing quark by $p^{\prime}$, that of the incoming quark by $p$ and the incoming gluon momenta by $k_{i}$, we use the following Fourier decomposition for the quark and gauge fields,

$$
\begin{aligned}
\psi(x) & =\int \frac{d^{4} p}{(2 \pi)^{4}} \psi(p) \mathrm{e}^{\mathrm{i} p \cdot x}, \\
\bar{\psi}(x) & =\int \frac{d^{4} p^{\prime}}{(2 \pi)^{4}} \bar{\psi}\left(p^{\prime}\right) \mathrm{e}^{-\mathrm{i} p^{\prime} \cdot x}, \\
A_{\mu}(x) & =\int \frac{d^{4} k_{i}}{(2 \pi)^{4}} A_{\mu}\left(k_{i}\right) \mathrm{e}^{\mathrm{i} k_{i} \cdot(x+a \hat{\mu} / 2)},
\end{aligned}
$$

where the momenta are restricted to the first Brillouin zone.

We use the standard realisation of the covariant derivatives acting to the right and to the left

$$
\begin{aligned}
\vec{D}_{\mu} \psi(x) & =\frac{1}{2 a}\left[U_{x, \mu} \psi(x+a \hat{\mu})-U_{x-a \hat{\mu}, \mu}^{\dagger} \psi(x-a \hat{\mu})\right] \\
\bar{\psi}(x) \overleftarrow{D}_{\mu} & =\frac{1}{2 a}\left[\bar{\psi}(x+a \hat{\mu}) U_{x, \mu}^{\dagger}-\bar{\psi}(x-a \hat{\mu}) U_{x-a \hat{\mu}, \mu}\right]
\end{aligned}
$$

The external ordinary derivative is taken as

$$
\partial_{\mu}(\bar{\psi} \cdots \psi)(x)=\frac{1}{a}[(\bar{\psi} \cdots \psi)(x+a \hat{\mu})-(\bar{\psi} \cdots \psi)(x)] .
$$

There are two convenient ways to define the corresponding operators in momentum space with non-zero momentum transfer $q$. One possibility is to "act" with $q$ at the lattice

point $x$. For the case of one covariant derivative this leads to (setting the Dirac matrix in the operator equal to the unit matrix for simplicity)

$$
\begin{aligned}
& \left(\bar{\psi} \stackrel{\leftrightarrow}{D}_{\mu} \psi\right)^{(I)}(q)=\sum_{x}\left(\bar{\psi} \stackrel{\leftrightarrow}{D}_{\mu} \psi\right)(x) \mathrm{e}^{i q \cdot x} \\
& \quad=\frac{1}{2 a} \sum_{x}\left[\bar{\psi}(x) U_{x, \mu} \psi(x+a \hat{\mu})-\bar{\psi}(x+a \hat{\mu}) U_{x, \mu}^{\dagger} \psi(x)\right]\left[\mathrm{e}^{i q \cdot x}+\mathrm{e}^{i q \cdot(x+a \hat{\mu})}\right]
\end{aligned}
$$

Alternatively, the momentum transfer can be applied to the "position center" (for the 
case of one covariant derivative $\vec{D}$ ) as follows

$$
\begin{array}{r}
\left(\bar{\psi} \vec{D}_{\mu} \psi\right)(q)=\frac{1}{2 a} \sum_{x}\left[\bar{\psi}(x) U_{x, \mu} \psi(x+a \hat{\mu}) \mathrm{e}^{i q \cdot(x+a \hat{\mu} / 2)}\right. \\
\left.-\bar{\psi}(x) U_{x-a \hat{\mu}, \nu}^{\dagger} \psi(x-a \hat{\mu}) \mathrm{e}^{i q \cdot(x-a \hat{\mu} / 2)}\right],
\end{array}
$$

which leads to

$$
\begin{aligned}
& \left(\bar{\psi} \stackrel{\leftrightarrow}{D}_{\mu} \psi\right)^{(I I)}(q) \\
& \quad=\frac{1}{a} \sum_{x}\left[\bar{\psi}(x) U_{x, \mu} \psi(x+a \hat{\mu})-\bar{\psi}(x+a \hat{\mu}) U_{x, \mu}^{\dagger} \psi(x)\right] \mathrm{e}^{i q \cdot(x+a \hat{\mu} / 2)}
\end{aligned}
$$

This realisation might be more suitable for numerical simulations. Note that

$$
\left(\bar{\psi} \stackrel{\leftrightarrow}{D}_{\mu} \psi\right)^{(I)}(q)=\cos \frac{a q_{\mu}}{2}\left(\bar{\psi} \stackrel{\leftrightarrow}{D}_{\mu} \psi\right)^{(I I)}(q)
$$

For the external ordinary derivative we use

$$
\begin{aligned}
\partial_{\mu}(\bar{\psi} \cdots \psi)(q) & =\frac{1}{a} \sum_{x}[(\bar{\psi} \cdots \psi)(x+a \hat{\mu})-(\bar{\psi} \cdots \psi)(x)] \mathrm{e}^{i q \cdot(x+a \hat{\mu} / 2)} \\
& =-\frac{2 i}{a} \sin \frac{a q_{\mu}}{2}(\bar{\psi} \cdots \psi)(q)
\end{aligned}
$$

The corresponding Feynman rules up to $O\left(g^{2}\right)$ derived from expressions based on (72) and (73) are marked by the superscripts (I) and (II), respectively.

$$
\begin{aligned}
\frac{O\left(g^{0}\right)}{\mathcal{O}_{\mu \nu \omega}^{(I I), D D}} & =\bar{\psi}\left(p^{\prime}\right) \gamma_{\mu} \psi(p) \frac{1}{a^{2}} \sin \frac{a\left(p+p^{\prime}\right)_{\nu}}{2} \sin \frac{a\left(p+p^{\prime}\right)_{\omega}}{2}, \\
\mathcal{O}_{\mu \nu \omega}^{(I), D D} & =\cos \frac{a\left(p-p^{\prime}\right)_{\nu}}{2} \cos \frac{a\left(p-p^{\prime}\right)_{\omega}}{2} \mathcal{O}_{\mu \nu \omega}^{(I I), D D}, \\
\mathcal{O}_{\mu \nu \omega}^{(I I), \partial D} & =\bar{\psi}\left(p^{\prime}\right) \gamma_{\mu} \psi(p) \frac{1}{a^{2}} \sin \frac{a\left(p-p^{\prime}\right)_{\nu}}{2} \sin \frac{a\left(p+p^{\prime}\right)_{\omega}}{2}, \\
\mathcal{O}_{\mu \nu \omega}^{(I), \partial D} & =\cos \frac{a\left(p-p^{\prime}\right)_{\omega}}{2} \mathcal{O}_{\mu \nu \omega}^{(I I), \partial D}, \\
\mathcal{O}_{\mu \nu \omega}^{\partial \partial \partial} & =\bar{\psi}\left(p^{\prime}\right) \gamma_{\mu} \psi(p) \frac{1}{a^{2}} \sin \frac{a\left(p-p^{\prime}\right)_{\nu}}{2} \sin \frac{a\left(p-p^{\prime}\right)_{\omega}}{2} .
\end{aligned}
$$


$\underline{O(g)}$

$$
\begin{aligned}
\mathcal{O}_{\mu \nu \omega}^{(I I), D D}= & g \sum_{\sigma} \bar{\psi}\left(p^{\prime}\right) \gamma_{\mu} A_{\sigma}\left(k_{1}\right) \psi(p) \cos \frac{a\left(p+p^{\prime}\right)_{\sigma}}{2} \\
& \times \frac{1}{a}\left[\delta_{\nu \sigma} \sin \frac{a\left(p+p^{\prime}-k_{1}\right)_{\omega}}{2}+\delta_{\omega \sigma} \sin \frac{a\left(p+p^{\prime}+k_{1}\right)_{\nu}}{2}\right], \\
\mathcal{O}_{\mu \nu \omega}^{(I), D D}= & \cos \frac{a\left(p-p^{\prime}+k_{1}\right)_{\nu}}{2} \cos \frac{a\left(p-p^{\prime}+k_{1}\right)_{\omega}}{2} \mathcal{O}_{\mu \nu \omega}^{(I I), D D}, \\
\mathcal{O}_{\mu \nu \omega}^{(I I), \partial D}= & g \bar{\psi}\left(p^{\prime}\right) \gamma_{\mu} A_{\omega}\left(k_{1}\right) \psi(p) \frac{1}{a} \sin \frac{a\left(p-p^{\prime}+k_{1}\right)_{\nu}}{2} \cos \frac{a\left(p+p^{\prime}\right)_{\omega}}{2} . \\
\mathcal{O}_{\mu \nu \omega}^{(I), \partial D}= & \cos \frac{a\left(p-p^{\prime}+k_{1}\right)_{\omega}}{2} \mathcal{O}_{\mu \nu \omega}^{(I I), \partial D} .
\end{aligned}
$$

$O\left(g^{2}\right)$ for the tadpole case $k_{2}=-k_{1}$

Here we restrict ourselves to the tadpole case $k_{2}=-k_{1}$ (cf. Fig. (2) as this is all we need in this paper. For general gluon momenta the Feynman rules are much more complicated.

$$
\begin{aligned}
\mathcal{O}_{\mu \nu \omega}^{(I I), D D}= & \frac{g^{2}}{2} \sum_{\sigma, \tau} \bar{\psi}\left(p^{\prime}\right) \gamma_{\mu} A_{\tau}\left(-k_{1}\right) A_{\sigma}\left(k_{1}\right) \psi(p) \\
& \times\left\{2 \delta_{\tau \nu} \delta_{\sigma \omega} \cos \frac{a\left(p+p^{\prime}+k_{1}\right)_{\nu}}{2} \cos \frac{a\left(p+p^{\prime}+k_{1}\right)_{\omega}}{2}\right. \\
& \left.-\left(\delta_{\tau \nu} \delta_{\sigma \nu}+\delta_{\tau \omega} \delta_{\sigma \omega}\right) \sin \frac{a\left(p+p^{\prime}\right)_{\nu}}{2} \sin \frac{a\left(p+p^{\prime}\right)_{\omega}}{2}\right\}, \\
\mathcal{O}_{\mu \nu \omega}^{(I), D D}= & \cos \frac{a\left(p-p^{\prime}\right)_{\nu}}{2} \cos \frac{a\left(p-p^{\prime}\right)_{\omega}}{2} \mathcal{O}_{\mu \nu \omega}^{(I I), D D}, \\
\mathcal{O}_{\mu \nu \omega}^{(I I), \partial D}= & -\frac{g^{2}}{2} \bar{\psi}\left(p^{\prime}\right) \gamma_{\mu} A_{\omega}\left(k_{1}\right) A_{\omega}\left(-k_{1}\right) \psi(p) \sin \frac{a\left(p-p^{\prime}\right)_{\nu}}{2} \sin \frac{a\left(p+p^{\prime}\right)_{\omega}}{2}, \\
\mathcal{O}_{\mu \nu \omega}^{(I), \partial D}= & \cos \frac{a\left(p-p^{\prime}\right)_{\omega}}{2} \mathcal{O}_{\mu \nu \omega}^{(I I), \partial D} .
\end{aligned}
$$

The Feynman rules for the operators with different Dirac matrices are obtained by the obvious replacements. Note that the forward case is realised for $O\left(g^{0}\right)$ and $O\left(g^{2}\right)$ (in the tadpole case) by $p=p^{\prime}$, for $O(g)$ we have to take $p^{\prime}=p+k_{1}$. 


\section{References}

[1] D. Müller, D. Robaschik, B. Geyer, F. M. Dittes and J. Horejsi, Fortsch. Phys. 42 (1994) 101;

X. D. Ji, Phys. Rev. Lett. 78 (1997) 610, Phys. Rev. D 55 (1997) 7114;

A. V. Radyushkin, Phys. Lett. B 380 (1996) 417, Phys. Lett. B 385 (1996) 333;

J. C. Collins, L. Frankfurt and M. Strikman, Phys. Rev. D 56 (1997) 2982.

[2] M. Diehl, Phys. Rept. 388 (2003) 41.

[3] A. Airapetian et al. [HERMES Collaboration], Eur. Phys. J. C 17 (2000) 389; Phys. Lett. B 535 (2002) 85;

S. Chekanov et al. [ZEUS Collaboration], Eur. Phys. J. C 24 (2002) 345.

[4] A. Airapetian et al. [HERMES Collaboration], Phys. Rev. Lett. 87 (2001) 182001;

S. Stepanyan et al. [CLAS Collaboration], Phys. Rev. Lett. 87 (2001) 182002;

C. Adloff et al. [H1 Collaboration], Phys. Lett. B 517 (2001) 47;

S. Chekanov et al. [ZEUS Collaboration], Phys. Lett. B 573 (2003) 46.

[5] X. Ji, J. Phys. G 24 (1998) 1181.

[6] P. Hägler, arXiv:hep-ph/0404138.

[7] M. Göckeler, R. Horsley, D. Pleiter, P. E. L. Rakow, A. Schäfer, G. Schierholz and W. Schroers [QCDSF Collaboration], Phys. Rev. Lett. 92 (2004) 042002.

[8] P. Hägler, J. Negele, D. B. Renner, W. Schroers, T. Lippert and K. Schilling [LHPC collaboration], Phys. Rev. D 68 (2003) 034505.

[9] M. Göckeler, P. Hagler, R. Horsley, D. Pleiter, P. E. L. Rakow, A. Schäfer, G. Schierholz, J. M. Zanotti, arXiv:hep-lat/0409162.

[10] M. Göckeler, R. Horsley, P. E. L. Rakow, H. Perlt, A. Schäfer, G. Schierholz and A. Schiller, arXiv:hep-lat/0409025.

[11] G.P. Lepage and S.J. Brodsky, Phys. Rev. D 22 (1980) 2157;

A.V. Efremov and A.V. Radyushkin, Theor. Math. Phys. 42 (1980) 97;

M.A. Shifman and M.I. Vysotsky, Nucl. Phys. B 186 (1981) 475.

[12] M. Göckeler, R. Horsley, E. M. Ilgenfritz, H. Perlt, P. Rakow, G. Schierholz and A. Schiller, Phys. Rev. D 54 (1996) 5705.

[13] M. Göckeler, unpublished notes. 
[14] H. Kawai, R. Nakayama and K. Seo, Nucl. Phys. B 189 (1981) 40.

[15] M. Göckeler, R. Horsley, E. M. Ilgenfritz, H. Perlt, P. E. L. Rakow, G. Schierholz and A. Schiller, Nucl. Phys. B 472 (1996) 309.

[16] S. Capitani, M. Göckeler, R. Horsley, H. Perlt, P. E. L. Rakow, G. Schierholz and A. Schiller, Nucl. Phys. B 593 (2001) 183.

[17] R. Horsley, H. Perlt, P. E. L. Rakow, G. Schierholz and A. Schiller, Nucl. Phys. B 693 (2004) 3.

[18] A. I. Davydychev and J. B. Tausk, Phys. Rev. D 53 (1996) 7381;

J. M. Campbell, E. W. N. Glover and D. J. Miller, Nucl. Phys. B 498 (1997) 397;

J. Fleischer, F. Jegerlehner and O. V. Tarasov, Nucl. Phys. B 672 (2003) 303. 